Del Arco Bravo, I., Flores Alarcia, O, y Silva, P. (2019). El desarrollo del modelo flipped classroom en la universidād: impacto de su implementación desde la voz del estudiantado. Revista de Investigación Educativa, 37(2), 451-469. DOI: http://dx.doi.org/10.6018/rie.37.2.327831

\title{
El desarrollo del modelo flipped classroom en la universidad: impacto de su implementación desde la voz del estudiantado
}

\section{The development of the model flipped classroom at university: impact of its implementation from student voice}

\author{
Isabel del Arco Bravo*, Óscar Flores Alarcia* y Patricia Silva García* \\ *Departamento de Pedagogía y Psicología. Facultad de Educación, Psicología y Trabajo Social. Universidad de Lleida
} (España)

\begin{abstract}
Resumen
El progreso del mundo digital ha generado un cambio radical en la manera de acceder $y$ transmitir el conocimiento. El aprendizaje ha dejado de ser una actividad interna e individual para pasar a ser una actividad colectiva y en red. Este artículo describe los resultados de un estudio cuantitativo que tuvo como objetivo analizar las percepciones y valoraciones de estudiantes de la Universidad de Lleida en relación con la implementación del modelo flipped classroom. Se aplicó un cuestionario diseñado ad hoc. Los datos se recogieron de manera longitudinal durante dos cursos académicos, 2015-2016 y 2016-2017. La muestra del estudio fue de 259 participantes. Los resultados muestran que mejoró la experiencia de aprendizaje y su efectividad, siendo los resultados más positivos en los estudiantes de mayor edad. De entre las conclusiones destacamos que este modelo genera una mayor motivación entre los estudiantes, pero al mismo tiempo mayores niveles de corresponsabilidad y compromiso en estudiantado y profesorado.

Palabras clave: modelos de enseñanza; estudiantes universitarios; prácticas de enseñanza; habilidades de estudio; flipped classroom.

Correspondencia: Óscar Flores Alarcia, oscar.flores@udl.cat, Universidad de Lleida, Facultad de Educación Psicología y Trabajo Social, Departamento de Pedagogía y Psicología, Av. de l’Estudi General, 4, Segon Pis, despatx 2.14, 25001, Lleida.
\end{abstract}




\begin{abstract}
The progress in the digital world has generated a radical change in the way to access and transmit knowledge. The learning process is no more an internal and individual activity; nowadays is a collective activity in the network. This article describes the results of a quantitative study which purpose was to analyse the students' perceptions and valuations regarding the implementations of the flipped classroom model. An ad hoc questionnaire was designed. Data were collected longitudinally during two academic years, 2015-2016 and 2016-2017. The sample of the study was 259 participants. Results show that in general learning experience and its effectivity improve, being the most positive results in the older students. Among the conclusions we highlight that the model improves students' motivation but it requires high levels of responsibility and commitment in both students and teachers.

Keywords: teaching models; university students; teaching practices; study skills; flipped classroom.
\end{abstract}

\title{
Introducción
}

El modelo flipped classroom pretende dar respuesta a la necesidad de replantear nuevos formatos metodológicos en los procesos formativos. La idea se basa en una reorganización del tiempo dentro y fuera del aula. Forma parte de un amplio movimiento pedagógico que se solapa con el aprendizaje basado en la investigación y resolución de problemas, enfoques que promueven la flexibilidad en los procesos educativos y la participación activa de los aprendices.

El origen lo encontramos en el año 2006 en los profesores de la Escuela Secundaria de Woodland Park en Colorado (Bergmann \& Sams, 2014). Aunque inicialmente la base era sustituir una explicación en el aula por un vídeo, el concepto ha evolucionado hacia una metodología que va mucho más lejos (Flores, 2015; Llamazares, 2014), donde se promueve una diversificación de recursos y el formador toma decisiones diferentes sobre el acto didáctico y la gestión de la relación con y entre los estudiantes (Pinnelli \& Fiorucci, 2015).

Hadman, McKnight, McKnight y Arfstrom (2013) han identificado que los modelos pedagógicos que transfieren el conocimiento teórico fuera del aula y utilizan el tiempo de clase para facilitar la adquisición y práctica de conocimientos cada vez son más recurrentes.

Van apareciendo evidencias acerca de su eficacia (Flores, Del Arco \& Silva, 2016; Tourón \& Santiago, 2015) aunque estos resultados no son aún definitivos ni garantes para generalizar la premisa de sus bondades (Goodwin \& Miller, 2014).

De acuerdo con Flores et al. (2016), la clase inversa pivota sobre tres ejes: las tareas que se realizan en el aula, la que se realizan fuera de ésta, y el proceso de evaluación (figura 1).

Una de las consecuencias de esta manera de desarrollar un proceso de enseñanza es el cambio de rol que se produce entre docente y discente, Silva (2015) afirma que:

El profesorado, con la aplicación de estrategias como la demostración activa, simulaciones, resolución de casos y exámenes entre pares, entre otros, ha generado un cambio en la actitud hacia la enseñanza (...). El protagonismo se traslada al estudiante y el rol 


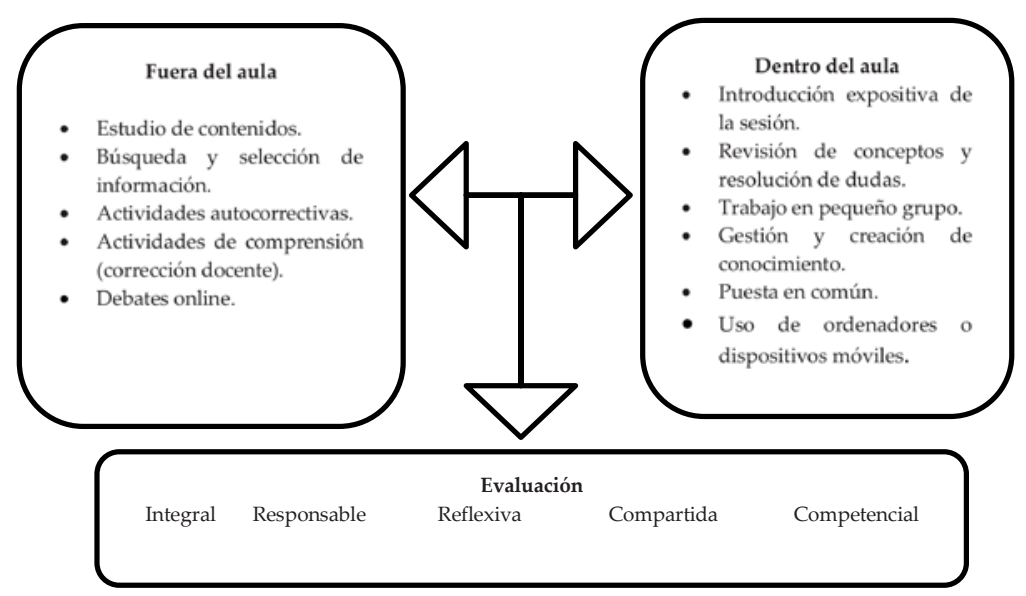

Figura 1. El modelo flipped classroom (Flores et al., 2016, p. 13).

del profesor cobra protagonismo como coordinador, moderador y facilitador de los temas y actividades (p. 9).

El modelo permite al profesorado una mayor flexibilidad para ofrecer en sus clases oportunidades de aprendizaje basadas en actividades de resolución de problemas, incidiendo en la retroalimentación y orientación a los estudiantes (Del Arco \& Camats, 2015) y reduciendo sustancialmente el tiempo que dedica a explicar contenidos "the flipped classroom is able to redesign a large lecture class into an active-learning class made of small groups" (Danker, 2015, p. 183).

Del Arco (2015), afirma que el estudiante desarrolla competencias como la selección de información, el trabajo cooperativo, el pensamiento crítico y la autoevaluación del aprendizaje. Se trata de potenciar la autonomía a través de métodos de enseñanza activos: “... donde el profesor se convierte en mediador, guía del proceso de aprendizaje y está abierto a la experimentación de diversas fórmulas metodológicas” (p. 5).

Este modelo supone una organización distinta en el tiempo y del espacio del trabajo diario (Hadman, McKnight, McKnight \& Arfstrom 2013). Modificar el ambiente de aprendizaje, utilizar mesas redondas, fomentar el trabajo en equipo y potenciar la comunicación son aspectos que consideramos nodales.

El aumento de protagonismo y de autonomía se potencia con el trabajo colaborativo que los alumnos deben realizar, en momentos presenciales y no presenciales (Shea \& Bidjerano, 2010), de manera desarrollamos un modelo formativo semipresencial en el que las tecnologías juegan un papel clave para que el proceso sea un éxito.

Así, por ejemplo, consideramos importante aprovechar herramientas tecnológicas que nos permiten:

- Ofrecer recursos en diferentes formatos (textuales, gráficos, audiovisuales, etc.).

- Desarrollar actividades de corrección automática, que ofrecen una gran variedad de posibilidades.

- Gestionar las actividades de comprensión con corrección docente de manera ágil. 
- Establecer espacios de colaboración y reflexión conjunta.

La falta de conocimientos o de formación no debe ser una excusa, como tampoco la idea erróneamente extendida de que los alumnos saben más que los profesores en herramientas TIC por el hecho de haber nacido en una sociedad digital (Flores \& Del Arco, 2013).

Sobre el proceso evaluativo, la apuesta es el desarrollo de una evaluación continuada durante todo el curso. El objetivo es conseguir una evaluación que se entrelace con el proceso de aprendizaje y que promueva la generación de mejores preguntas y del aprendizaje a partir de los errores (Tourón \& Santiago, 2015).

Cómo de observa en la figura 1, y de acuerdo con Flores et al. (2016), consideramos que la evaluación a través de este modelo debe ser:

- Integral, englobando el trabajo fuera y dentro del aula.

- Responsable, dejando que una parte de las actividades de evaluación las realice el alumno sin la mediación directa del profesor.

- Reflexiva, incluyendo un trabajo de análisis y síntesis de la información.

- Compartida, atendiendo a una coevaluación entre compañeros.

- Competencial, con actividades y prácticas no centradas en la memorística, sino en la resolución de supuestos prácticos.

Cabiscol (2015) indica que este modelo permite integrar desde el primer momento procesos autorreguladores de evaluación, aportando aspectos como el aumento de la oportunidad de recibir feedback con respecto a la manera de llegar a los conocimientos adquiridos por los alumnos, el respeto al ritmo de aprendizaje y la mejora de su implicación y autonomía.

Estudios como el realizado por Díez Ochoa (2016) apuntan que la clase inversa mejora el rendimiento de los estudiantes comparando los resultados en exámenes finales del mismo nivel de dificultad. También aparecen evidencias acerca del aumento del interés y la motivación hacía las materias de estudio (McCallum, Schultz, Sellke \& Spartz, 2015).

En la misma línea se expresan Hernández-Nanclares y Pérez-Rodríguez (2015), Danker (2015), Larsen (2015), Flores (2015), Flores et al. (2016) y Chih-Yuan y Wu (2016), coincidiendo en que tras la aplicación de este modelo los estudiantes muestran una actitud positiva hacia las materias de estudio y el aprendizaje, conectan nuevas ideas con aprendizajes previos y se mejoran los resultados académicos.

La investigación presenta los resultados de un estudio cuantitativo desarrollado en la Universidad de Lleida los cursos 2015-16 y 2016-2017 a partir de la aplicación del método de aula invertida en asignaturas de salud y educación.

Nos interesaba sobretodo profundizar en aspectos concretos del proceso de enseñanza-aprendizaje: el rol docente y discente, el uso de las TIC, el proceso de evaluación y la autonomía.

El objetivo general era analizar las percepciones y valoraciones de los estudiantes ante la aplicación del modelo flipped classroom. Se platearon cuatro objetivos específicos:

- Identificar si el alumnado percibe que con el aula invertida se produce un cambio de rol docente y discente. 
- Conocer su valoración sobre si el modelo favorece la integración de las TIC en el proceso de enseñanza-aprendizaje.

- Analizar su valoración acerca del proceso de evaluación competencial.

- Averiguar si perciben este modelo como una propuesta que promueve su autonomía.

\section{Método}

El mundo educativo vive inmerso en una época de cambios que reclaman una renovación del sistema, desde una perspectiva organizativa y metodológica. El problema es que muchas veces los cambios que se implementan se realizan sin tener en cuenta el punto de vista de quienes serán sus destinatarios: los estudiantes.

Nuestra motivación es aproximarnos a la aplicación del modelo de clase inversa y conocer cuáles son los impactos de su implementación profundizando desde la voz del estudiantado (Díaz Pareja, 2002; Levin, 2000; Martínez Álvarez, 2011; Mitra, 2005; Rodríguez Romero, 2008; Rojas, Haya \& Lázaro-Visa, 2012; Rudduck, 2007; Rudduck \& McIntyre, 2007) para conocer sus percepciones y valoraciones.

Compartimos la idea de la voz del estudiantado a partir de la definición de Nieto y Portela (2008) "la voz que se recorre para aglutinar aportaciones heterogéneas que diferentes autores vienen haciendo para conceptuar la relevancia que tiene realzar el papel que pueden desarrollar los alumnos en las actividades educativas y las organizaciones en que estas tienen lugar" (p. 2).

El estudio que se presenta se centra en una metodología de corte cuantitativo con cuestionario único post-test.

La muestra está configurada entre los estudiantes de las asignaturas de la Universidad de Lleida (UdL), donde se aplica el modelo flipped.

Los datos se recogieron de manera longitudinal durante dos cursos académicos, 2015-2016 y el 2016-2017 entre aquellos estudiantes matriculados en asignaturas donde se aplicó el modelo flipped, tal y como se muestra en la tabla 1. La población está constituida por 265 estudiantes.

Tabla 1

Población total de estudiantes matriculados en las tres asignaturas analizadas

\begin{tabular}{|c|c|c|c|}
\hline $\begin{array}{l}\text { Alumnos } \\
\text { Asignaturas }\end{array}$ & $\begin{array}{c}\text { Alumnos } \\
\text { matriculados } \\
2015-16\end{array}$ & $\begin{array}{c}\text { Alumnos } \\
\text { matriculados } \\
2016-17\end{array}$ & Total \\
\hline $\begin{array}{l}\text { Investigación e innovación psicopedagógica (Más- } \\
\text { ter en Psicopedagogía) }\end{array}$ & 31 & 28 & 59 \\
\hline $\begin{array}{l}\text { Procesos didácticos y organizativos (Grado de } \\
\text { Educación Infantil y Primaria) }\end{array}$ & 66 & 66 & 132 \\
\hline Educación para la salud (Grado de Fisioterapia) & 38 & 36 & 74 \\
\hline Total & 135 & 130 & 265 \\
\hline
\end{tabular}


La muestra unificada y válida de sujetos que participaron en el estudio fue de 259, en la que se aprecia una gran mayoría de mujeres: 198, el 76.4\% (IC: 70.8\% - 81.5\% con una confianza del $95 \%$ ) frente a los varones (61; un $23.6 \%$ ). La edad se presentaba categorizada en el cuestionario y considerada esta manera: un $90.7 \%$ (235 participantes; IC: $86.5 \%$ - 94.0\%) ha señalado encontrarse en el rango de menores de 25 años. El resto se ubica en el rango igual/más de 25 años (tabla 2). En cuanto a las titulaciones, aunque hay mayoría de participantes de Magisterio (168; 64.9\% con IC: 58.7\% - 70.7\%) las otras dos titulaciones están bien representadas: Fisioterapia con 53 participantes (20.5\%) y Psicopedagogía con 38 (14.7\%).

Tabla 2

Análisis descriptivo. Muestra de participantes ( $\mathrm{N}=259$ casos válidos)

\begin{tabular}{lccccc}
\hline \multirow{2}{*}{ Variable } & Categoría & Número de & & \multicolumn{2}{c}{ I.C. 95\% } \\
\cline { 5 - 6 } & casos & \% & Lim. Inf. & Lim. Sup. \\
\hline Género & Mujeres & 198 & 76.4 & 70.8 & 81.5 \\
& Hombres & 61 & 23.6 & 18.5 & 29.2 \\
Edad & Menos de 25 & 235 & 90.7 & 86.5 & 94.0 \\
& De 25 a 30 & 17 & 6.6 & 3.9 & 10.3 \\
\multirow{2}{*}{ Titulación } & Más de 30 & 7 & 2.7 & 1.1 & 5.5 \\
& Magisterio & 168 & 64.9 & 58.7 & 70.7 \\
& Fisioterapia & 53 & 20.5 & 15.7 & 25.9 \\
& Psicopedagogía & 38 & 14.7 & 10.6 & 19.6 \\
\hline
\end{tabular}

Los datos se recogieron a través de un cuestionario ad-hoc, compuesto por 19 ítems de formato Likert con 5 opciones de respuesta desde $1=$ total desacuerdo, hasta $5=$ total acuerdo. El cuestionario presenta ítems vinculados con cuatro dimensiones (variables dependientes) que despliegan los objetivos específicos formulados:

- El cambio de rol docente y discente.

- La integración de las TIC en el proceso de enseñanza-aprendizaje.

- El desarrollo del proceso de evaluación propuesto.

- El desarrollo de la autonomía.

En referencia a la dimensión del cambio de rol docente y discente, las preguntas se centraron en si los estudiantes observaron con la metodología de la clase inversa un cambio real en sus actuaciones y en las del profesorado, indicando si las dinámicas de comunicación eran distintas y si cambiaba su percepción sobre su protagonismo en el proceso formativo respecto a metodologías tradicionales. 
Sobre la integración de las TIC, el cuestionario presentaba una serie de ítems para que los estudiantes indicaran si el modelo favorecía su integración en el proceso de enseñanza-aprendizaje, y si además facilitaban las tareas y la interacción.

Para la parte del proceso de evaluación, se formularon ítems vinculados a identificar su opinión sobre aspectos como: una evaluación no centrada en la memorización, la coevaluación entre iguales como alternativa, la evaluación fuera del aula sin mediación docente, etc. También se les pedía si consideraban que el conocimiento adquirido era mayor que en el caso que la asignatura se hubiera desarrollado bajo un modelo formativo tradicional.

Finalmente, sobre el desarrollo de la autonomía el cuestionario presentaba una serie de preguntas vinculadas al respeto por la autonomía y ritmos de aprendizaje de cada estudiante y el grado de seguridad sentida durante el desarrollo de la asignatura. Estas dimensiones se analizaron considerando como variables independientes el género, la edad y la titulación.

La validación del cuestionario se realizó en dos fases:

Primeramente, se realizó una validación de contenido por expertos (10 jueces) para conocer la pertinencia y adecuación de los ítems de acuerdo al objetivo del estudio y teniendo en cuenta los destinatarios.

En una segunda fase se procedió a una validación factorial de constructo, que incluyó un análisis exploratorio y descriptivo de los ítems y un análisis factorial exploratorio. Para el análisis exploratorio de los ítems se han empleado los índices de asimetría y curtosis, junto al Test de bondad de ajuste de Kolmogorov-Smirnov de bondad de ajuste al modelo de la curva normal de Gauss y donde se consideran significativos solo los desvíos graves del modelo, es decir al 1\% ( $p<.01 ; p<.010$ en tres decimales). Para el análisis descriptivo se han empleado los estadísticos habituales: media, mediana, desviación estándar, rango (valor mínimo/máximo) y amplitud intercuartil.

Del análisis descriptivo y exploratorio se descubre una validez del constructo para la muestra con la que se trabaja (>200 casos) y una buena variabilidad en todos los ítems.

La fiabilidad del instrumento se alcanza mediante la ecuación "alfa" de Cronbach (diseño de consistencia interna o método intraclase). El valor obtenido es muy elevado:.884 (IC al 95\%:.862 -.903; en escala 0-1) indicando una fiabilidad muy buena por parte de esta muestra en el instrumento diseñado.

Para el tratamiento estadístico de los datos las técnicas y test estadísticos empleados han sido:

- Coeficientes de correlación de Pearson y Spearman, entre pares de variables cuantitativas.

- Test de diferencia de medias en grupos independientes entre sí: T de Student y Anova. Alternativas no paramétricas respectivas: Test de Mann-Whitney y Test de Kruskal-Wallis.

- Test de Friedman para la comparación de valores promedio en muestras relacionadas.

- El nivel de significación fijado es el habitual 5\% (significativo si $p \leq .05 ; p \leq .050$ en tres decimales). 


\section{Resultados}

Presentamos a continuación los resultados y su análisis descriptivo atendiendo a las dimensiones consideradas:

\section{Dimensión: Cambio de rol profesor/alumno}

Los resultados indican (tabla 3) que las respuestas de los sujetos se concentran en los valores del 4 al 5 , indicando por tanto una posición tendente más al acuerdo hacia lo que propone el contenido de cada ítem. Por ello, las medias tienen valores elevados (entre 3.90 y 4.20) a la par que se caracterizan por una cierta asimetría negativa (más valores altos) que las aleja significativamente del modelo de la campana normal de Gauss ( $p<.001$ en el Test de bondad de ajuste de Kolmogorov-Smirnov). Se aplicó el Test de Friedman para el contraste de la diferencia entre las medias de unos y otros ítems, encontrándose diferencias altamente significativas $(p<.001)$ de manera que podemos admitir que el mayor grado de acuerdo se ha manifestado hacia el contenido del ítem 3 y el menor hacia los ítems no 1 y 4 . El coeficiente de fiabilidad de esta dimensión fue:.844 (IC al 95\%:.812 -.872) según la ecuación "alfa" de Cronbach, es decir que debe ser considerado como muy bueno.

Tabla 3

Análisis descriptivo. Ítems de la dimensión: Cambio de rol profesor/alumno (N=259). Fiabilidad:.844

\begin{tabular}{ccccccccccc}
\hline & \multicolumn{4}{c}{ \% de respuesta de cada opción } & \multicolumn{2}{c}{ Descriptivos } & \multicolumn{2}{c}{ Forma } & Test KS \\
\cline { 2 - 11 } Ítem & $\mathbf{1}$ & $\mathbf{2}$ & $\mathbf{3}$ & $\mathbf{4}$ & $\mathbf{5}$ & Media & $\begin{array}{c}\text { Desv. } \\
\text { Est. }\end{array}$ & Asimetría & Curtosis & P-sig \\
\hline № 1 & 1.5 & 7.3 & 17.8 & 45.9 & 27.4 & 3.90 & 0.94 & -0.80 & 0.40 & $.000^{* *}$ \\
№ 2 & 0.8 & 6.2 & 18.1 & 36.3 & 38.6 & 4.06 & 0.94 & -0.79 & 0.01 & $.000^{* *}$ \\
№ 3 & 0.8 & 3.9 & 12.7 & 40.2 & 42.5 & 4.20 & 0.86 & -1.05 & 0.99 & $.000^{* *}$ \\
№ 4 & -- & 6.2 & 17.0 & 46.7 & 30.1 & 4.01 & 0.85 & -0.63 & -0.13 & $.000^{* *}$ \\
№ 5 & 3.9 & 9.3 & 14.7 & 34.0 & 38.2 & 3.93 & 1.12 & -0.95 & 0.11 & $.000^{* *}$ \\
\hline
\end{tabular}

Nota. ${ }^{*}=$ Desvío grave significativo $(\mathrm{p}<.01)$ la variable no se ajusta a la normalidad; Test de Friedman: Chi2=23.41; $p=.001$

Así, los estudiantes encuestados manifiestan un alto acuerdo en considerar que con el modelo flipped se produce un cambio real en el rol del estudiante y del profesorado. Sin embargo, aparece un cierto margen de desacuerdo en sus opiniones cuando se considera que este modelo ofrece buenas oportunidades de comunicación con el profesorado, no todos están totalmente de acuerdo en esta afirmación. Además, creen que las orientaciones del profesorado no siempre han servido para mejorar los conceptos de la asignatura. 
En referencia a la relación de esta dimensión con las variables independientes, en relación al género no se observaron diferencias significativas (tabla 4).

Tabla 4

Análisis diferencial. Significación de la diferencia entre las medias de la dimensión Cambio de rol docente/discente en función del Género

\begin{tabular}{lcccccc}
\hline Variable & $\begin{array}{c}\text { Mujeres } \\
(\mathbf{n}=\mathbf{1 9 8})\end{array}$ & $\begin{array}{c}\text { Hombres } \\
(\mathbf{n}=61)\end{array}$ & T Student & \multicolumn{2}{c}{ Mann-Whitney } \\
\cline { 2 - 7 } & $\begin{array}{c}\text { Media } \\
\text { (D.E.) }\end{array}$ & $\begin{array}{c}\text { Media } \\
\text { (D.E.) }\end{array}$ & Valor & p-valor & Valor & p-valor \\
\hline $\begin{array}{l}\text { Cambio de } \\
\text { rol docente/ } \\
\text { discente }\end{array}$ & $4.04(0.74)$ & $3.96(0.76)$ & 0.76 & $.451 \mathrm{NS}$ & 0.77 & $.440 \mathrm{NS}$ \\
\hline
\end{tabular}

Nota. N.S. $=$ NO significativo $(p>.05)$

Sobre las diferencias en esta dimensión por razón de edad, se observó que las medias eran significativamente superiores en los estudiantes de edades a partir de los 25 años (tabla 5).

Tabla 5

Análisis diferencial. Significación de la diferencia entre las medias de la dimensión Cambio de rol docente/discente en función de la Edad

\begin{tabular}{ccccccc}
\hline Variable & $\begin{array}{c}\text { Menores } \\
\text { de 25 años } \\
(\mathbf{n}=235)\end{array}$ & $\begin{array}{c}\text { Mayor/Igual } \\
\text { a 25 años } \\
(\mathbf{n}=24)\end{array}$ & T Student & Mann-Whitney \\
\cline { 2 - 7 } & $\begin{array}{c}\text { Media } \\
\text { (D.E.) }\end{array}$ & Media (D.E.) & Valor & p-valor & Valor & p-valor \\
\hline $\begin{array}{c}\text { Cambio de } \\
\text { rol docente/ } \\
\text { discente }\end{array}$ & $3.99(0.72)$ & $4.27(0.87)$ & -1.78 & .077 NS & -2.53 & $.011 *$ \\
\hline
\end{tabular}

Nota . N.S. $=$ NO significativo $\left(\mathrm{p}>\right.$.05) $;{ }^{*}=$ Significativo al $5 \%(p \leq .05) ;{ }^{* *}=$ Altamente significativo al $1 \%(p<.01)$

Finalmente, la diferencia de esta dimensión con la variable titulación mostró que los sujetos con puntuaciones más altas son los de la titulación en Psicopedagogía. Les siguen los que estudian Magisterio y por último los de Fisioterapia (tabla 6). 
Tabla 6

Análisis diferencial. Significación de la diferencia entre las medias de variables de la dimensión Cambio de rol docente/discente en función de la Titulación

\begin{tabular}{lccccccc}
\hline Variables & $\begin{array}{c}\text { Magisterio } \\
(\mathbf{n}=168)\end{array}$ & $\begin{array}{c}\text { Psicopedagogía } \\
(\mathbf{n}=38)\end{array}$ & $\begin{array}{c}\text { Fisioterapia } \\
(\mathbf{n}=53)\end{array}$ & Anova 1 f.e.f. & \multicolumn{2}{c}{$\begin{array}{c}\text { Kruskal- } \\
\text { Wallis }\end{array}$} \\
\cline { 2 - 8 } & Media (D.E.) & Media (D.E.) & Media (D.E.) & Valor & p-sig & Valor & p-sig \\
\hline $\begin{array}{l}\text { Cambio de } \\
\text { rol docen- } \\
\text { te/discente }\end{array}$ & $3.97(0.73)$ & $4.54(0.32)$ & $3.81(0.84)$ & 12.79 & $.000^{* *}$ & 25.78 & $.000^{* *}$ \\
\hline
\end{tabular}

Nota. ${ }^{* *}=$ Altamente significativo al $1 \%(p<.01)$

Se constata que son los estudiantes de mayor edad, que también coinciden con la titulación de máster, los que manifiestan que el cambio de rol profesor/estudiante se hace evidente con este modelo pedagógico.

\section{Dimensión: Integración de las TIC en el proceso de enseñanza-aprendizaje}

De nuevo las respuestas de los sujetos se agrupan en los valores del extremo superior de la escala (tabla 7), indicando por ello una opinión tendente hacia el acuerdo. Las medias alcanzan valores elevados siendo las distribuciones asimétricas negativas, lo que las desvía significativamente del modelo normal de Gauss ( $p<.001$ en el Test KS de bondad de ajuste). Según el Test de Friedman para el contraste de la diferencia entre estos ítems, existen diferencias significativas $(p<.01)$ por lo que podemos admitir que el mayor grado de acuerdo se ha manifestado hacia el contenido los ítems ítem 8 y 9 y el menor hacia el no 7. El grado de fiabilidad alcanzado en este caso es alto:.852 (IC al 95\%:.821 -.879).

Tabla 7

Análisis descriptivo. Ítems de la dimensión: Integración de las TIC (N=259). Fiabilidad:.852

\begin{tabular}{lccccccccccc}
\hline & \multicolumn{3}{c}{ \% de respuesta de cada opción } & \multicolumn{2}{c}{ Descriptivos } & \multicolumn{2}{c}{ Forma } & \multirow{2}{*}{$\begin{array}{c}\text { Test } \\
\text { KS }\end{array}$} \\
\cline { 2 - 9 } Ítem & $\mathbf{1}$ & $\mathbf{2}$ & $\mathbf{3}$ & $\mathbf{4}$ & $\mathbf{5}$ & $\begin{array}{c}\text { Me- } \\
\text { dia }\end{array}$ & $\begin{array}{c}\text { Desv. } \\
\text { Est. }\end{array}$ & Asimetría & Curtosis & P-sig \\
\hline № 6 & -- & 1.5 & 17.4 & 45.9 & 35.1 & 4.15 & 0.75 & -0.47 & -0.44 & $.000^{* *}$ \\
№ 7 & 0.4 & 1.9 & 18.9 & 49.8 & 29.0 & 4.05 & 0.77 & -0.55 & 0.33 & $.000^{* *}$ \\
№ 8 & -- & 2.3 & 15.1 & 44.0 & 38.6 & 4.19 & 0.77 & -0.64 & -0.14 & $.000^{* *}$ \\
№ 9 & 0.4 & 3.5 & 13.1 & 39.4 & 43.6 & 4.22 & 0.83 & -0.97 & 0.64 & $.000^{* *}$ \\
№ 10 & -- & 3.5 & 15.8 & 45.9 & 34.7 & 4.12 & 0.80 & -0.64 & -0.06 & $.000^{* *}$ \\
\hline
\end{tabular}

Nota. ${ }^{* *}=$ Desvío grave significativo $(p<.01)$ la variable no se ajusta a la normalidad; Test de Friedman: Chi2=17.21; $p=.002$ 
A opinión de los estudiantes la integración de las TIC es una realidad en el modelo flipped, y su utilización facilita la colaboración entre compañeros. Sin embargo, no existe un alto consenso en afirmar que su uso sea un factor determinante para facilitar el aprendizaje.

Cuando analizamos esta dimensión correlacionando con las variables independientes vemos que no existen diferencias significativas por razón de edad y género, pero si por titulación, en que aparecen diferencias significativas entre las valoraciones de los alumnos de Psicopedagogía en relación a las otras dos titulaciones (tabla 8).

Tabla 8

Análisis diferencial. Significación de la diferencia entre las medias de la dimensión Integración de las TIC en función de la Titulación

\begin{tabular}{lccccccc}
\hline Variables & $\begin{array}{c}\text { Magisterio } \\
(\mathbf{n}=168)\end{array}$ & $\begin{array}{c}\text { Psicopedagogía } \\
(\mathbf{n}=\mathbf{3 8})\end{array}$ & $\begin{array}{c}\text { Fisioterapia } \\
(\mathbf{n}=53)\end{array}$ & Anova 1 f.e.f. & Kruskal-Wallis \\
\cline { 2 - 7 } & Media (D.E.) & Media (D.E.) & Media (D.E.) & Valor & p-sig & Valor & p-sig \\
\hline $\begin{array}{l}\text { Integra- } \\
\text { ción de } \\
\text { las TIC }\end{array}$ & $4.13(0.62)$ & $4.45(0.51)$ & $4.00(0.64)$ & 6.35 & $.002^{* *}$ & 12.87 & $.002^{* *}$ \\
\hline
\end{tabular}

Nota. ${ }^{* *}=$ Altamente significativo al $1 \%(p<.01)$

Volvemos a encontrar estas diferencias en alumnos de máster que ya tienen un recorrido formativo y una mayor capacidad sobre el aporte que estas herramientas pueden hacer a la docencia

\section{Dimensión: Proceso de evaluación}

Una vez más las respuestas se acumulan en los valores del extremo superior de la escala (tabla 9) correspondientes al acuerdo, incluso en este caso en bastantes de los ítems se concentran más en el valor 5. Por ello las medias presentan valores elevados con distribuciones que son muy asimétricas negativas y desvíos significativos de la campana normal ( $p<.001$ en el Test KS). El Test de Friedman nos indica que existen diferencias altamente significativas $(p<.0001)$ tales que el mayor grado de acuerdo se ha dirigido hacia el contenido del ítem 11 y el menor hacia el $\mathrm{n}^{\mathrm{o}} 15$. El grado de fiabilidad alcanzado es bueno, aunque algo inferior a las anteriores:.736 (IC al 95\%:.682 -.782).

Se constata que los estudiantes valoran muy positivamente que la evaluación se base en actividades y prácticas no centradas en la memorización, sino en la resolución de supuestos prácticos. Aplicar diferentes técnicas de evaluación es muy bien valorado por los estudiantes, que también admiten que el modelo permite desarrollarse competencialmente, en cuanto que son capaces de aplicar lo aprendido a diferentes situaciones fuera de las trabajadas en el aula. 
Tabla 9

Análisis descriptivo. Ítems de la dimensión: Proceso de evaluación (N=259). Fiabilidad:.736

\begin{tabular}{|c|c|c|c|c|c|c|c|c|c|c|}
\hline \multirow[b]{2}{*}{ Ítem } & \multicolumn{5}{|c|}{ \% de respuesta de cada opción } & \multicolumn{2}{|c|}{ Descriptivos } & \multicolumn{2}{|c|}{ Forma } & \multirow{2}{*}{$\begin{array}{c}\text { Test } \\
\text { KS } \\
\text { P-sig }\end{array}$} \\
\hline & $\begin{array}{c}1 \\
\text { (MD) }\end{array}$ & $\begin{array}{c}2 \\
\text { (D) }\end{array}$ & 3 & $\begin{array}{c}4 \\
\text { (A) }\end{array}$ & $\begin{array}{c}5 \\
\text { (MA) }\end{array}$ & Media & $\begin{array}{c}\text { Desv. } \\
\text { Est. }\end{array}$ & Asimetría & Curtosis & \\
\hline № 11 & 0.8 & 1.2 & 3.9 & 28.2 & 66.0 & 4.58 & 0.70 & -2.11 & 5.95 & $.000^{* *}$ \\
\hline № 12 & 0.8 & 1.9 & 11.2 & 39.0 & 47.1 & 4.30 & 0.80 & -1.18 & 1.64 & $.000^{* *}$ \\
\hline № 13 & 1.5 & 5.4 & 13.9 & 33.6 & 45.6 & 4.16 & 0.96 & -1.12 & 0.80 & $.000^{* *}$ \\
\hline № 14 & 1.2 & 4.6 & 8.5 & 44.8 & 40.9 & 4.20 & 0.86 & -1.26 & 1.80 & $.000^{* *}$ \\
\hline № 15 & 4.6 & 7.7 & 16.6 & 37.5 & 33.6 & 3.88 & 1.10 & -0.94 & 0.27 & $.000^{* *}$ \\
\hline № 16 & 1.2 & 4.6 & 19.3 & 40.9 & 34.0 & 4.02 & 0.91 & -0.79 & 0.37 & $.000^{* *}$ \\
\hline
\end{tabular}

Nota. ${ }^{* *}=$ Desvío grave significativo $(p<.01)$ la variable no se ajusta a la normalidad; Test de Friedman: Chi2=124.09; $p<.0000$

No se observaron diferencias significativas en esta dimensión con la variable del género. Respecto a la variable edad se mantiene la tendencia que los alumnos de más edad puntúan más alto con las afirmaciones propuestas (tabla 10), aunque en este caso no de manera significativa.

Tabla 10

Análisis diferencial. Significación de la diferencia entre las medias de la dimensión Proceso de evaluación en función de la Edad

\begin{tabular}{lccccccc}
\hline Variables & $\begin{array}{c}\text { Menores } \\
\text { de 25 años } \\
(\mathbf{n}=235)\end{array}$ & $\begin{array}{c}\text { Mayor/ } \\
\text { Igual a } \\
\mathbf{2 5} \text { años } \\
(\mathbf{n = 2 4 )}\end{array}$ & T Student & Mann-Whitney \\
\cline { 2 - 8 } & $\begin{array}{c}\text { Media } \\
\text { (D.E.) }\end{array}$ & $\begin{array}{c}\text { Media } \\
(\text { D.E. })\end{array}$ & Valor & p-valor & Valor & p-valor \\
\hline $\begin{array}{l}\text { Proceso de } \\
\text { evaluación }\end{array}$ & $4.18(0.55)$ & $4.26(0.89)$ & -0.60 & $.548 \mathrm{NS}$ & -1.86 & $.063 \mathrm{NS}$ \\
\hline
\end{tabular}

Nota. N.S. $=$ NO significativo $(p>.05) ;{ }^{*}=$ Significativo al $5 \%(p<.05) ; * *=$ Altamente significativo al $1 \%(p<.01)$

Finalmente, en relación con la variable titulación (tabla 11) sí se encontraron diferencias estadísticamente muy significativas $(p<.01)$. Siguiendo la tendencia de las otras dimensiones, los sujetos con puntuaciones más altas son los de Psicopedagogía. Les siguen los que estudian Magisterio y por último los de Fisioterapia.

Los test por pares nos indican que entre estas las titulaciones de Magisterio y Fisioterapia ofrecen una casi significación $(p<.10)$ en esta dimensión. 
Tabla 11

Análisis diferencial. Significación de la diferencia entre las medias de la dimensión Proceso de evaluación en función de la Titulación

\begin{tabular}{lcccccccc}
\hline \multirow{2}{*}{ Variables } & $\begin{array}{c}\text { Magisterio } \\
(\mathbf{n}=\mathbf{1 6 8})\end{array}$ & $\begin{array}{c}\text { Psicopedagogía } \\
(\mathbf{n}=38)\end{array}$ & $\begin{array}{c}\text { Fisioterapia } \\
(\mathbf{n}=53)\end{array}$ & Anova 1 f.e.f. & \multicolumn{2}{c}{ Kruskal-Wallis } \\
\cline { 2 - 7 } & $\begin{array}{c}\text { Media } \\
(\text { D.E. })\end{array}$ & Media (D.E.) & Media (D.E.) & Valor & p-sig & Valor & p-sig \\
\hline $\begin{array}{l}\text { Proceso de } \\
\text { evaluación }\end{array}$ & $4.19(0.56)$ & $4.47(0.33)$ & $3.99(0.74)$ & 7.67 & $.001^{* *}$ & 13.13 & $.001^{* *}$ \\
\hline
\end{tabular}

Nota. $^{* *}=$ Altamente significativo al $1 \%(p<.01)$

Vuelve a destacarse que son los estudiantes de máster los que valoran mejor el proceso de evaluación desarrollado en el modelo, por encima de los estudiantes de grado.

\section{Dimensión: Promoción de la autonomía}

Se mantiene las distribuciones asimétricas negativas con desvíos significativos con respecto a la normal ( $p<.001$ en el Test KS). En este caso, en los ítems 17 y 19 las respuestas se concentran en los valores del extremo del acuerdo por lo que sus medias son elevadas (tabal 12).

El ítem 18 está enunciado en sentido inverso a los demás, puesto que una respuesta favorable habla de un mayor grado de inseguridad hacia la asignatura (en tanto que los demás ítems hablan de seguridad e interés hacia ella). Por ello, para una correcta interpretación de las respuestas, este ítem debe ser recodificado a la inversa. Así, en el ítem 18, al ser recodificado, aparecen porcentajes tendentes más hacia el lado del desacuerdo, de modo que su media es menor. Por esto el test de Friedman nos permite concluir que las diferencia entre ellas son estadísticamente significativas $(p<.001)$. La fiabilidad alcanzada es, esta vez, escasa:.441 (IC al 95\%:.312 -.550).

Tabla 12

Análisis descriptivo. Ítems de la dimensión: Promoción de la autonomía (N=259). Fiabilidad::441

\begin{tabular}{|c|c|c|c|c|c|c|c|c|c|c|}
\hline \multirow[b]{2}{*}{ Ítem } & \multicolumn{5}{|c|}{$\%$ de respuesta de cada opción } & \multicolumn{2}{|c|}{ Descriptivos } & \multicolumn{2}{|c|}{ Forma } & \multirow{2}{*}{$\begin{array}{c}\text { Test } \\
\text { KS } \\
\text { P-sig }\end{array}$} \\
\hline & $\begin{array}{c}1 \\
\text { (MD) }\end{array}$ & $\begin{array}{c}2 \\
\text { (D) }\end{array}$ & 3 & $\begin{array}{c}4 \\
\text { (A) }\end{array}$ & $\begin{array}{c}5 \\
\text { (MA) }\end{array}$ & $\begin{array}{c}\text { Me- } \\
\text { dia }\end{array}$ & $\begin{array}{c}\text { Desv. } \\
\text { Est. }\end{array}$ & Asimetría & Curtosis & \\
\hline № 17 & 0.4 & 2.3 & 25.5 & 42.1 & 29.7 & 3.98 & 0.83 & -0.39 & -0.34 & $.000^{* *}$ \\
\hline № $18 \_R$ & 11.6 & 31.7 & 25.9 & 21.6 & 9.3 & 2.85 & 1.16 & 0.20 & -0.85 & $.000^{* *}$ \\
\hline № 19 & 3.1 & 9.3 & 20.5 & 34.4 & 32.8 & 3.85 & 1.08 & -0.74 & -0.15 & $.000^{* *}$ \\
\hline
\end{tabular}

Nota. ${ }^{* *}=$ Desvío grave significativo $(p<.01)$ la variable no se ajusta a la normalidad; Test de Friedman: Chi2=112.25; $p<.000$ 
Los estudiantes encuestados valoraron muy positivamente que el modelo facilite la adaptación del proceso de enseñanza a los diferentes ritmos de aprendizaje. El nivel de inseguridad al inicio de la asignatura parece ser una sensación percibida y vivida por los estudiantes ante nuevos planteamientos de trabajo. Sin embargo, el interés y la seguridad van incrementándose a lo largo del desarrollo de la asignatura.

En esta dimensión no existen diferencias significativas en relación con el género, pero sí respecto a la edad (tabla 13), a favor de los estudiantes de mayor edad, siendo las diferencias significativas en esta dimensión $(p<.01)$.

\section{Tabla 13}

Análisis diferencial. Significación de la diferencia entre las medias de la dimensión Promoción de la autonomía en función de la Edad

\begin{tabular}{lcccccc}
\hline Variables & $\begin{array}{c}\text { Menores } \\
\text { de 25 años } \\
(\mathbf{n = 2 3 5 )}\end{array}$ & $\begin{array}{c}\text { Mayor/ } \\
\text { Igual a 25 } \\
\text { años (n=24) }\end{array}$ & T Student & \multicolumn{2}{c}{ Mann-Whitney } \\
\cline { 2 - 7 } & $\begin{array}{c}\text { Media } \\
\text { (D.E.) }\end{array}$ & $\begin{array}{c}\text { Media } \\
\text { (D.E.) }\end{array}$ & Valor & p-valor & Valor & p-valor \\
\hline $\begin{array}{l}\text { Promoción de } \\
\text { la autonomía }\end{array}$ & $3.53(0.55)$ & $3.89(0.64)$ & -3.03 & $.003^{* *}$ & -2.61 & $.009^{* *}$ \\
\hline
\end{tabular}

Nota. N.S. $=$ NO significativo $(p>.05) ;{ }^{*}=$ Significativo al $5 \%(p \leq .05) ;{ }^{* *}=$ Altamente significativo al $1 \%(p<.01)$

Por último, en relación con la variable titulación, se mantiene la diferencia estadísticamente muy significativa de la misma forma que en las anteriores: los sujetos con puntuaciones más altas son los de Psicopedagogía. Les siguen los que estudian Magisterio y por último los de Fisioterapia (tabla 14).

Los test por pares nos indican que entre estas las titulaciones de Magisterio y Fisioterapia ofrecen una casi significación $(p<.10)$ en esta dimensión.

\section{Tabla 14}

Análisis diferencial. Significación de la diferencia entre las medias de la dimensión Promoción de la autonomía en función de la Titulación

\begin{tabular}{lccccccc}
\hline \multirow{2}{*}{ Variables } & $\begin{array}{c}\text { Magisterio } \\
(\mathbf{n}=168)\end{array}$ & $\begin{array}{c}\text { Psico-peda- } \\
\text { gogía }(\mathbf{n}=38)\end{array}$ & $\begin{array}{c}\text { Fisioterapia } \\
(\mathbf{n}=53)\end{array}$ & Anova 1 f.e.f. & Kruskal-Wallis \\
\cline { 2 - 7 } & Media (D.E.) & $\begin{array}{c}\text { Media } \\
\text { (D.E.) }\end{array}$ & Media (D.E.) & Valor & p-sig & Valor & p-sig \\
\hline $\begin{array}{l}\text { Promoción } \\
\text { de la } \\
\text { autonomía }\end{array}$ & $3.53(0.54)$ & $3.92(0.57)$ & $3.40(0.53)$ & 10.97 & $.000^{* *}$ & 19.97 & $.000^{* *}$ \\
\hline
\end{tabular}

Nota. ${ }^{* *}=$ Altamente significativo al $1 \%(p<.01)$ 


\section{Discusión}

El modelo Flipped Classroom se ha visto como una oportunidad de experimentar con otros sistemas de enseñanza y aprendizaje. En las sesiones de clase se ofrecieron más opciones de aprendizaje basadas en actividades centradas en la resolución de problemas, estudio de casos, uso del tiempo de trabajo fuera de clase, el trabajo en equipo, entre otras. La metodología fue aceptada con agrado, aunque se reconoce que al principio costó entrar en la dinámica. Del mismo modo que destaca Llamazares (2014) el protagonismo se trasladó a los estudiantes y el rol del profesor fue más de coordinación, moderación y facilitador de los temas y actividades desarrolladas tanto fuera del aula como dentro.

La metodología proporcionó más oportunidades para involucrarse en su propio aprendizaje. Según los resultados expuestos, se modificaron los esquemas de enseñanza y ha permitido interactuar con sus pares y aprender de ellos. El trabajo en equipo, por ejemplo, es algo que se valoró de forma significativa. De acuerdo con Hadman, McKnight, McKnight y Arfstrom (2013), los estudiantes han sido el centro de aprendizaje, el profesorado promovió una participación activa en su formación y la evaluación de su propio aprendizaje utilizando diferentes recursos y herramientas para evaluar.

En dos de los tres casos, los estudiantes mencionaron la necesidad de mayor coordinación y comunicación entre el profesorado. La Organización para la Cooperación y el Desarrollo Económicos (OCDE, 2008) recomienda avanzar hacia un liderazgo cada vez más sostenible y distribuido entre diferentes personas y los futuros profesionales evidentemente lo han de desarrollar por lo que creemos es una evidencia de su eficacia (Tourón \& Santiago, 2015).

Identificamos que la autonomía es un elemento clave que se enriquece con este modelo, coincidimos con Shea y Bidjerano (2010) pues como señalan el estudiante ha sido el centro del trabajo en las Asignaturas y ha movilizado el desarrollo de competencias. De la misma manera se ratifica lo expuesto por Silva (2015) que señala que los alumnos son los protagonistas y el trabajo en grupos promueve un trabajo más cooperativo ya que les permite interactuar más y mejor aprovechando las experiencias y el conocimiento de cada uno.

Los resultados coinciden con el Clintondale High School de Michigan, donde la tasa de fracaso de los estudiantes de matemáticas de 9o grado disminuyó del 44 al 13\% después de adoptar el Modelo (Finkel, 2012). En la misma línea Yarbro, Arfstrom, McKnight y McKnight (2014) y Moraros, Islam, Yu, Banow y Schindelka (2015) recogen diversos estudios de casos en diferentes etapas y niveles educativos y los resultados apuntan hacia una mejora en el rendimiento de los estudiantes y un aumento de su satisfacción y la de los profesores.

\section{Conclusiones}

El modelo Flipped classroom en la Universidad supone una alternativa para generar cambios en los roles a desempeñar por los estudiantes y los docentes.

El estudio muestra que los estudiantes se implican en el cambio de actuaciones que ellos deben desempeñar y que su nivel de seguridad en su desempeño es mayor a medida que avanza la asignatura. La transmisión y el consumo de información, como 
nos anuncia Prieto (2017), se realiza fuera de clase y se convierten en tareas a desarrollar de forma autónoma por los estudiantes. Se reservan, para el tiempo de clase, aquellas tareas que realmente precisan de la presencia del profesor. Bergmann y Sams (2012) nos anuncian, y así hemos podido corroborar, que lo más relevante de este modelo en relación a los estudiantes es que éstos desarrollan un rol activo, interactuando en los grupos colaborativos que se establecen.

Esta nueva forma de hacer es percibida por los estudiantes con interés, aumentando la motivación por la asignatura. Valoran muy positivamente la oportunidad de integrar las tecnologías y la posibilidad de desarrollar competencias aplicables en diferentes contextos profesionales, sin embargo, no creen que las TIC sean el elemento clave y único para facilitar los aprendizajes. En el caso de los estudiantes de educación valoran el conocer nuevas técnicas didácticas que ayudan a su desarrollo competencial docente en un futuro, técnicas que vayan más allá del uso exclusivo de las TIC.

Nuestro estudio deja entrever que el rol del profesorado, que se convierte en mediador de los procesos de aprendizaje, no es siempre percibido así por los estudiantes. Las orientaciones de los docentes no siempre les han ayudado a asimilar mejor los conceptos. Ello nos lleva a pensar en lo consolidados que están determinados modelos docentes y lo difícil que resulta este cambio de rol. Para los estudiantes la figura del profesor en este modelo cobra relevancia, como mediador, asesor y guía (Sánchez Vera, Solano Rodríguez \& González Calatayud, 2016). Es preciso una formación del profesorado universitario para consolidar los nuevos roles que se le asignan en modelos pedagógico-didáctico como el Flipped.

Este modelo exige a los estudiantes que traigan mejor preparada las clases y además han de ser conscientes de sus dudas respecto a los contenidos trabajados, pero también, a los docentes les exige conocer los problemas de comprensión y errores conceptuales de los alumnos, para activar mecanismos de apoyo y orientación en el proceso de aprendizaje.

Los estudiantes de máster, que coinciden con los de mayor edad, son los que manifiestan unas opiniones más favorables hacia este modelo formativo. Ello nos lleva a considerar que los estudiantes llegan a los estudios universitarios sin tener desarrolladas las habilidades para trabajar en el aula con un rol activo y participante. Los de grado siguen instalados en un rol pasivo y receptor con dificultades para desarrollar un rol proactivo. Podríamos considerar que este modelo es más efectivo con estudiantes de niveles o etapas superiores, pero ello no impide renunciar a su aplicación en niveles inferiores por lo que ayudaría a formar estudiantes autónomos y proactivos en su propio proceso de aprendizaje.

Otro apartado a tener en cuenta es la evaluación. Los estudiantes han manifestado su total acuerdo por la substitución de procesos evaluadores centrados en la memorización por otros basados en la aplicación práctica y en la resolución de supuestos. Se constata la adecuación de fórmulas de evaluación competencial donde los estudiantes demuestren, individualmente o en grupo, la adquisición de una serie de habilidades que les hacen competentes en uno u otro ámbito del conocimiento.

Los estudiantes participantes manifiestan una mayor aceptación por el cambio en los procesos evaluativos, pero no saben justificar si los resultados de aprendizaje son significativamente superiores a los obtenidos con una metodología tradicional. 
La percepción que tienen es que no existe tanta diferencia, sin embargo, este dato es fácilmente contratable con los resultados objetivos de evaluación y con una evaluación diferida para contrastar la permanencia y fijación de las habilidades desarrolladas.

Para concluir, este modelo genera una mayor motivación entre los estudiantes, pero al mismo tiempo mayores niveles de corresponsabilidad y compromiso entre los protagonistas del proceso de enseñanza-aprendizaje.

\section{Referencias}

Bergmann, J., \& Sams, A. (2012). Flip your classroom: Reach every student in every class every day. Eugene, OR, EE.UU.: International Society for Technology in Education.

Bergmann, J., \& Sams, A. (2014). Dale la vuela a tu clase. Lleva tu clase a cada estudiante, en cualquier momento y cualquier lugar. Madrid: Ediciones SM.

Cabiscol, A. (2015). La evaluación como reguladora del proceso de aprendizaje en la clase invertida. Escuela, 11-12.

Chih-Yuan, J., \& Wu, Y. T. (2016). Analysis of learning achievement and teacher-student interactions in flipped and conventional classrooms. International Review of Research in Open and Distributed Learning, 17(1), 79-99. doi: https://doi.org/10.19173/irrodl. v17i1.2116

Danker, B. (2015). Using Flipped Classroom Approach to Explore Deep Learning in Large Classrooms. IAFOR Journal of Education, 3(1), 171-186. Recuperado de https:// eric.ed.gov/?id=EJ1100618

Del Arco, I. (2015). "Flippear", siendo alumno digital, desde el Trabajo cooperativo. Escuela, 5-6.

Del Arco, I., \& Camats, R. (2015). La formación de maestros en "flipped classroom". Escuela, 16-17.

Díaz Pareja, E. M. (2002). Importancia de la "voz del alumno" para descubrir sus necesidades. Enseñanza, 20, 281-299.

Díez Ochoa, A. (2016, 23 de noviembre). Cómo invertir tu clase con éxito [Sitio web]. Recuperado de http://www.theflippedclassroom.es/como-invertir-tu-clase-con-exito/

Finkel, E. (2012). Flipping the Script in K-12. District Administration, 48(10), 28-34. Recuperado de https://eric.ed.gov/?id=EJ984780

Flores, O., \& Del Arco, I. (2013). Nativos digitales, inmigrantes digitales: rompiendo mitos. Un estudio sobre el dominio de las TIC en profesorado y estudiantado de la Universidad de Lleida. Bordón, 65(2), 59-74. doi: https://doi.org/10.13042/ brp.2013.65204

Flores, O. (2015). Planificar la "flipped classroom". Escuela, 7-8.

Flores, O., Del Arco, I., \& Silva, P. (2016). The flipped classroom model at the university: analysis based on professors' and students' assessment in the educational field. International Journal of Educational Technology in Higher Education, 13(21), 1-12. doi: https://doi.org/10.1186/s41239-016-0022-1

Goodwin, B., \& Miller, K. (2013). Research Says / Evidence on Flipped Classrooms Is Still Coming in. Technology-Rich Learning, 70(6), 78-80. Recuperado de http://www.ascd. org/publications/educational-leadership/mar13/vol70/num06/Evidence-on-FlippedClassrooms-Is-Still-Coming-In.aspx 
Hadman, N., McKnight, P. E., McKnight, K., \& Arfstrom, K. M. (2013). The flipped learning model: A white paper based on the literature review titled. Flipped Learning Network, 3-20. Recuperado de http://researchnetwork.pearson.com/wpcontent/ uploads/WhitePaper_FlippedLearning.pdf

Hernández-Nanclares, N., \& Pérez-Rodríguez, M. (2015). Students' Satisfaction with a Blended Instructional Design: The Potential of "Flipped Classroom" Higher Education. Journal of Interactive Media in Education, 1(4), 1-12. doi: http://doi.org/10.5334/jime.397

Larsen, J. (2015). Adult Students' Experiences of a Flipped Mathematics Classroom. Adults Learning Mathematics. An International Journal, 10(1), 50-67. Recuperado de http://files.eric.ed.gov/fulltext/EJ1077724.pdf

Levin, B. (2000). Putting students at the center in education reform. Journal of Educational Change, 1(2), 155-172.

Llamazares, A. (2014). Algunas reflexiones en torno al blended-learning. El Guiniguada. Revista de investigaciones y experiencias en Ciencias de la Educación, 23, 63-70. Recuperado de http://ojsspdc.ulpgc.es/ojs/index.php/ElGuiniguada/article/view/249/227

Martínez Álvarez, D. B. (2011). La voz de los estudiantes. Experiencias en torno a la escuela. CPU-e. Revista de Investigación Educativa, 12, 1-10. Recuperado de http://www. redalyc.org/html/2831/283121721009/

McCallum, S., Schultz, J., Sellke, K., \& Spartz, J. (2015). An Examination of the Flipped Classroom Approach on College Student Academic Involvement. International Journal of Teaching and Learning in Higher Education, 27(1), 42-55.

Moraros, J., Islam, A., Yu, S., Banow, R., \& Schindelka, B. (2015). Flipping for success: evaluating the effectiveness of a novel teaching approach in a graduate level setting. BMC Medical Education, 15(27). doi: https://doi.org/10.1186/s12909-015-0317-2

Mitra, D. L. (2005). Adults advising youth: leading while getting out of the way. Educational Administration Quarterly, 41(3), 520-553.

Nieto, J. M., \& Portela, A. (2008). La inclusión de la voz del alumnado en el asesoramiento para la mejora de las prácticas educativas. Profesorado. Revista de currículum y formación del profesorado, 12, 1, 1-15.

Organización para la Cooperación y el Desarrollo Económicos [OCDE] (2008). Improving school leadership, Volume I. Practice and Policy. Paris: Autor.

Pinnelli, S., \& Fiorucci, A. (2015). University and Flipped Learning TICEDIL Project: Framework and Design. Comunicación presentada en el 12th International Conference on Cognition and Exploratory Learning in Digital Age (CELDA), 24 - 26 de octubre (p. 217-224), Maynooth, Greater Dublin (Irlanda). Recuperado de http://files.eric.ed.gov/ fulltext/ED562195.pdf

Prieto, A (2017, 25 de julio). Flipped classroom ¿Cuáles son sus ventajas? ¿Cuál es su origen y su evolución posterior? ¿Por qué no es una moda más? ¿Por qué mejora el aprendizaje? ¿Por qué deberías leer sobre este modelo en este verano? [Sitio web]. Recuperado de http:// profesor3punto0.blogspot.com.es/2016/07/flipped-classroom-cuales-son-sus_7.html

Rudduck, J. (2007). Student voice, sudent engagement, and school reform. En D. Thiessen, \& A. Cook-Sather (Eds.) International handbook of student experience in elementary and secondary school (p. 587-610). Dordrecht, Países Bajos: Springer.

Rudduck, J., \& McIntyre, D. (2007). Improving learning through consulting pupils. London, UK: Routledge. 
Rodríguez Romero, M. M. (2008). El asesoramiento, el poder del profesorado y la voz del alumnado. Profesorado. Revista de currículum y formación del profesorado, 12(1), 1-15.

Rojas, S., Haya, I., \& Lázaro-Visa, S. (2012). La voz del alumnado en la mejora escolar: niños y niñas como investigadores en Educación Primaria. Revista de Educación, 359, 81-101.

Sánchez Vera, M. M., Solano Rodríguez, I. M., \& González Calatayud, V. (2016). FLIPPED-TIC: Una experiencia de Flipped Classroom con alumnos de Magisterio. RELATEC Revista Latinoamericana de Tecnología Educativa, 15(3), 69-81. doi: https://doi. org/10.17398/1695-288X.15.3.69

Shea, P., \& Bidjerano, T. (2010). Learning presence: Towards a theory of self-efficacy, selfregulation, and the development of a communities of inquiry in online and blended learning environments. Computers \& Education, 55(4), 1721-1731. doi: https://doi. org/10.1016/j.compedu.2010.07.017

Silva, P. (2015). "Flipped classroom" y aprendizaje activo: el rol docente. Escuela, 9-10.

Tourón, J., \& Santiago, R. (2015). El modelo Flipped Learning y el desarrollo del talento en la escuela. Revista de Educación, 368, 196-231. Recuperado de https://goo.g1/O9dB98

Yarbro, J., Arfstrom, K. M., McKnight, K., \& McKnight, P. (2014). Extension of a Review of Flipped Learning. Flipped Learning Network, 2-17. Recuperado de https://flippedlearning.org/wp-content/uploads/2016/07/Extension-of-FLipped-Learning-LIt-ReviewJune-2014.pdf

Fecha de recepción: 13 de abril de 2018.

Fecha de revisión: 23 de abril de 2018.

Fecha de aceptación: 15 de abril de 2019. 
